\title{
Ionic imaging and bio-energetic analysis of club drug-induced cognitive deficiency by time-of-flight secondary ion mass spectrometry (TOF-SIMS)
}

\author{
F.-D. Mai ${ }^{1 *}$, S.-C. Youn ${ }^{2,3}$ L.-Y. Chen ${ }^{3}$, R.-J. Chiou ${ }^{4}$, T.-J. Lai², W.-C. Liao ${ }^{3}$, \\ H.-M. Chang ${ }^{4}$ \\ ${ }^{1}$ Department of Biochemistry and Molecular Cell Biology, School of Medicine, College of Medicine, \\ Taipei Medical University, Taipei, Taiwan \\ ${ }^{2}$ Institute of Medicine, College of Medicine, Chung Shan Medical University, Taichung, Taiwan \\ ${ }^{3}$ Department of Anatomy, Faculty of Medicine, Chung Shan Medical University, Taichung, Taiwan \\ ${ }^{4}$ Department of Anatomy and Cell Biology, School of Medicine, College of Medicine, Taipei Medical \\ University, Taipei, Taiwan \\ *e-mail:fordmai@tmu.edu.tw
}

Key words: gamma-hydroxybutyric acid (GHB), cognitive dysfunction, hippocampal, TOF-SIMS

Motivation and Aim: Excessive exposure to club drug gamma-hydroxybutyric acid (GHB) would cause cognitive dysfunction in which impaired hippocampal $\mathrm{Ca}^{2+}$-mediated neuroplasticity may correlate with this deficiency. However, the potential changes of in vivo $\mathrm{Ca}^{2+}$ together with molecular machinery engaged in GHB-induced cognitive dysfunction have never been reported. This study aims to determine these changes in bio-energetic level through ionic imaging, spectrometric, biochemical, morphological, as well as behavioral approaches.

Methods and Algorithms: Adolescent rats subjected to GHB were processed for TOFSIMS, immunohistochemistry, biochemical assay, together with Morris water maze to detect the ionic, molecular, neurochemical, and behavioral changes of GHB-induced cognitive dysfunction, respectively. Extent of oxidative stress and bioenergetics were assessed by levels of lipid peroxidation, $\mathrm{Na}^{+} / \mathrm{K}^{+}$ATPase, cytochrome oxidase, and $\left[{ }^{14} \mathrm{C}\right]-$ 2-deoxyglucose activity.

Results: The study indicated that in GHB intoxicated rats, decreased $\mathrm{Ca}^{2+}$ imaging and reduced NMDAR1, nNOS, and p-CREB reactivities were detected in hippocampus. Depressed $\mathrm{Ca}^{2+}$-mediated signaling corresponded well with intense oxidative stress, diminished $\mathrm{Na}^{+} / \mathrm{K}^{+}$ATPase, reduced COX, and decreased 2-DG activity, which all contributes to the development of cognitive deficiency. As impaired $\mathrm{Ca}^{2+}$-mediated signaling and oxidative stress significantly contribute to GHBinduced cognitive dysfunction, delivering agent(s) that improves hippocampal bio-energetics may thus serve as a promising strategy to counteract the club drug-induced cognitive dysfunction emerging in our society nowadays.

Conclusion: In summary, with the assistance of advanced spectrometric, ionic imaging, biochemical, morphological as well as behavioral approaches, the present study addressed for the first time that chronic and excessive exposure to GHB would cause cognitive dysfunction in which impaired hippocampal bio-energetics may contribute to the pathogenesis of this deficiency.

Acknowledgements: This study is supported by the research grants from the Taipei Medical University (TMU101-AE1-B13) and the Taipei Medical University Hospital (104TMU-TMUH-09), Taipei, Taiwan.

\section{References}

1. Chen L.Y. et al. (2017) Melatonin successfully rescues hippocampal bioenergetics and improves cognitive function following drug intoxication by promoting Nrf2-ARE signaling activity. J. Pineal Res. 2017; 63:e12417. 